\title{
FOURIER SERIES AND MEAN MODULI OF CONTINUITY*
}

\author{
BY \\ OTTO SZÁSZ
}

1. Introduction. Let $f(\theta)$ be a real-valued function of the real variable $\theta$, of period $2 \pi$, and let $f(\theta) \varepsilon L . \dagger$ We consider the formal Fourier series of $f(\theta)$ and write

$$
\begin{aligned}
f(\theta) & \sim \sum_{n=-\infty}^{\infty} c_{n} e^{i n \theta} \sim a_{0} / 2+\sum_{n=1}^{\infty}\left(a_{n} \cos n \theta+b_{n} \sin n \theta\right), \\
c_{n} & =\frac{1}{2 \pi} \int_{-\pi}^{\pi} f(\tau) e^{-i n \tau} d \tau=\frac{1}{2}\left(a_{n}-i b_{n}\right), c_{-n}=\overline{c_{n}}, n=0,1,2, \cdots .
\end{aligned}
$$

We denote by $\operatorname{Lip}(\alpha)$ the class of functions satisfying the condition

$$
|f(\theta+t)-f(\theta)|<K|t|^{\alpha},
$$

where $\alpha>0$ and $K=K_{f}$ is a constant depending on $f$.

In 1914, S. Bernstein [1, 2] $\ddagger$ proved the following result:

If $f(\theta) \varepsilon \operatorname{Lip}(\alpha), \alpha>1 / 2$, then $\sum\left|c_{n}\right|<\infty$; for $\alpha<1 / 2$ this is no longer true.

This result was generalized by the writer [12] in two directions:

1. The class $\operatorname{Lip}(\alpha)$ was replaced by the more general class

$\operatorname{Lip}_{2}(\alpha ; 2) \quad\left\{\frac{1}{2 \pi} \int_{-\pi}^{\pi}[f(\theta+2 t)+f(\theta-2 t)-2 f(\theta)]^{2} d \theta\right\}^{1 / 2}<K t^{\alpha}, \quad t>0$.

2. The exponent of convergence of the sequence of Fourier coefficients $\left\{c_{n}\right\}$ of a function of class $\operatorname{Lip}_{2}(\alpha ; 2)$ was determined, that is, the smallest constant $k$ such that $\sum\left|c_{n}\right|^{k+\epsilon}<\infty$ for all $\epsilon>0$. It was proved that $k=2 /(2 \alpha+1)$ for $0<\alpha \leqq 1$ and that there exist functions in $\operatorname{Lip}_{2}(\alpha ; 2)$ such that $\sum\left|c_{n}\right|^{2 /(2 \alpha+1)}=\infty$.

In a later paper [14] these results were extended to more general classes,

$\operatorname{Lip}_{2}(\alpha ; p) \quad\left\{\frac{1}{2 \pi} \int_{-\pi}^{\pi}|f(\theta+2 t)+f(\theta-2 t)-2 f(\theta)|{ }^{p} d \theta\right\}^{1 / p}<K t^{\alpha}$

$$
t>0,0<\alpha \leqq 1,1<p \leqq 2 .
$$

* Presented to the Society, December 31, 1936; received by the editors December 29, 1936.

The author is indebted to Professor J. D. Tamarkin for many valuable suggestions in the process of writing this paper.

$\dagger$ That is, $f(\theta)$ is integrable in the sense of Lebesgue. In general, by $L^{p}$ we denote the class of functions measurable and such that $|f(\theta)| p_{\varepsilon} L$.

$\ddagger$ Numbers in brackets refer to the bibliography at the end of this paper. 
For functions of this class, the exponent of convergence of the sequence $\left\{c_{n}\right\}$ was found to be $k=p /(\alpha p+p-1)$ and it was proved that there exist functions in $\operatorname{Lip}_{2}(\alpha ; p)$ such that $\sum\left|c_{n}\right|^{k}=\infty$.

In an earlier paper Hardy and Littlewood [5] found the same value for $k$ for the class

$\operatorname{Lip}_{1}(\alpha ; p) \quad\left\{\frac{1}{2 \pi} \int_{-\pi}^{\pi}|f(\theta+t)-f(\theta-t)|^{p} d \theta\right\}^{1 / p}<K t^{\alpha}, \quad t>0$,

under the restrictive assumption $\alpha p>1 .^{*}$

Let $f(\theta)$ be continuous and of period $2 \pi$. The modulus of continuity of $f(\theta)$ is defined by

$$
\xi(f ; \delta) \equiv \xi(\delta)=\max _{|t| \leqq \delta} \max _{\theta}|f(\theta+t)-f(\theta)| .
$$

In 1934, S. Bernstein [3] generalized his result as follows:

If $\sum_{1}^{\infty} n^{-1 / 2} \xi(1 / n)<\infty$ then $\sum\left|c_{n}\right|<\infty$. Conversely let $\eta(\delta)$ be a positive function such that $\eta(\delta) \delta^{-\alpha}$ is increasing in $\delta$ for sufficiently small positive values of $\alpha$, and decreasing for some $\alpha<1$. If in addition $\sum_{1}^{\infty} n^{-1 / 2} \eta(1 / n)=\infty$ then there exists a continuous function $f(\theta)$ such that $\xi(f ; \delta)<\eta(\delta)$ while $\sum\left|c_{n}\right|=\infty$.

In what follows we shall prove some theorems which include all the results mentioned above as special cases and which also extend certain results of Hardy and Littlewood concerning the classes $\operatorname{Lip}_{1}(\alpha ; p)$. $\dagger$

The following facts will be frequently used in the sequel.

(i) Young-Hausdorff inequalities. Let $1<p \leqq 2, g(\theta) \sim \sum_{-\infty}^{\infty} \gamma_{n} e^{i n \theta}$. Then

$$
\begin{aligned}
&\left\{\sum\left|\gamma_{n}\right|^{p^{\prime}}\right\}^{1 / p^{\prime}} \leqq\left\{\frac{1}{2 \pi} \int_{-\pi}^{\pi}|g(\tau)|^{p} d \tau\right\}^{1 / p}, \ddagger \\
&\left\{\frac{1}{2 \pi} \int_{-\pi}^{\pi}|g(\tau)|^{p^{\prime}} d \tau\right\}^{1 / p^{\prime}} \leqq\left\{\sum\left|\gamma_{n}\right|^{p}\right\}^{1 / p} .
\end{aligned}
$$

* This paper of Hardy and Littlewood contains many important results concerning the class $\operatorname{Lip}_{1}(\alpha ; p) ;$ cf. also [4]. It should be observed that the class $\operatorname{Lip}_{2}(\alpha ; p)$ includes the class $\operatorname{Lip}_{1}(\alpha ; p)$.

$\dagger$ Results analogous to the theorem above of S. Bernstein were obtained for the convergence of Fourier integrals by Titchmarsh [15] and by Hille and Tamarkin [8]. Results of the present paper can also be extended to the cases of Fourier integrals and of almost periodic functions. These extensions will be treated in a subsequent paper. The main results of the present paper were communicated to the Harvard Mathematical Colloquium on May 23, 1935.

$\ddagger$ By $p^{\prime}, q^{\prime}, \cdots$ we denote quantities which are related to $p, q, \cdots$ by $1 / p+1 / p^{\prime}=1 / q+1 / q^{\prime}$ $=\cdots=1$. 
It should be observed that Young-Hausdorff inequalities are trivially satisfied even in the case $p=1$, if we agree to replace

$$
\left\{\sum\left|\gamma_{n}\right|^{p^{\prime}}\right\}^{1 / p^{\prime}}, \quad\left\{\frac{1}{2 \pi} \int_{-\pi}^{\pi}|g(\tau)|^{p^{\prime}} d \tau\right\}^{1 / p^{\prime}}
$$

by $\sup _{n}\left|\gamma_{n}\right|, \sup _{\tau}|g(\tau)|$ respectively, where $\sup _{\tau}|g(\tau)|$ denotes in this case the "effective upper bound of $|g(\tau)|$ " [7, p. 135].

(ii) Hölder inequality. Assuming $q>1$, we have

$$
\begin{gathered}
\sum_{\nu=1}^{\infty}\left|a_{\nu} b_{\nu}\right| \leqq\left(\sum_{\nu=1}^{\infty}\left|a_{\nu}\right|^{q}\right)^{1 / q}\left(\sum_{\nu=1}^{\infty}\left|b_{\nu}\right|^{q^{\prime}}\right)^{1 / q^{\prime}} \\
\int_{a}^{b}|f(t) g(t)| d t \leqq\left(\int_{a}^{b}|f(t)|^{q} d t\right)^{1 / q}\left(\int_{a}^{b}|g(t)|^{q^{\prime}} d t\right)^{1 / q^{\prime}}
\end{gathered}
$$

with the same agreement as in (i) in case $q=1$.

(iii) Minkowski inequalities. If $q \geqq 1$,

$$
\begin{gathered}
\left\{\int_{a}^{b} d t\left(\int_{\alpha}^{\beta}|\phi(t, \tau)| d \tau\right)^{q}\right\}^{1 / q} \leqq \int_{\alpha}^{\beta} d \tau\left(\int_{a}^{b}|\phi(t, \tau)|^{q} d t\right)^{1 / q}, \\
\left\{\int_{a}^{b}\left|\sum_{\nu=1}^{n} \phi_{\nu}(x)\right|^{q} d x\right\}^{1 / q} \leqq \sum_{\nu=1}^{n}\left\{\int_{a}^{b}\left|\phi_{\nu}(x)\right|^{q} d x\right\}^{1 / q} .
\end{gathered}
$$

(iv) If we put

$$
\frac{1}{b-a}\left(\int_{a}^{b}|\phi(t)|^{p} d t\right)^{1 / p}=M_{p}(\phi ; a, b) \equiv M_{p}(\phi) \equiv M_{p}, \quad p>0,
$$

then $M_{p}$ is an increasing* function of $p$ and $\log \left(M_{p}\right)^{p}$ is a convex function of $p$, that is

$$
\begin{gathered}
\left(M_{p}\right)^{p} \leqq\left(M_{p_{1}}\right)^{\rho_{1} p_{1}}\left(M_{p_{2}}\right)^{\rho_{2} p_{2}}, \quad p=\rho_{1} p_{1}+\rho_{2} p_{2}, \quad \rho_{1}+\rho_{2}=1, \\
\rho_{1} \geqq 0, \quad \rho_{2} \geqq 0 .
\end{gathered}
$$

Furthermore, on denoting by $M(\phi ; a, b) \equiv M(\phi) \equiv M$ the effective upper bound of $|\phi|$ in $(a, b)$ we have

$$
\lim _{p \rightarrow \infty} M_{p}(\phi) \equiv M_{\infty}(\phi)=M(\phi) .
$$

Finally if we allow $p_{2} \rightarrow \infty$ in (1.3) and observe that then

$$
\rho_{1}=\frac{p_{2}-p}{p_{2}-p_{1}} \rightarrow 1, \quad \rho_{2} p_{2}=\frac{p-p_{1}}{p_{2}-p_{1}} p_{2} \rightarrow p-p_{1},
$$

* By increasing (decreasing) we mean non-decreasing (non-increasing), unless explicitly stated to the contrary. 
we have

$$
\left(M_{p}\right)^{p} \leqq\left(M_{p_{1}}\right)^{p_{1}} M^{p-p_{1}}, \quad 0<p_{1} \leqq p .^{*}
$$

The notation $\eta(t)$ will be used to denote a positive decreasing function of $t$ such that $\eta(t) \downarrow 0$ as $t \downarrow 0$. By $\gamma$ will be meant an arbitrary positive constant. The symbol $O(\phi(t, \gamma))$ will have its usual meaning with the understanding that $O$ refers to $\phi$ as a function of $t$, for $\gamma$ fixed, and that $O$ need not hold uniformly for all $\gamma$.

2. Auxiliary propositions. In order not to interrupt the subsequent discussion we shall prove in this section some inequalities which will be of fundamental importance in the sequel.

LEMMA 2.1. Let $0 \leqq A_{1} \leqq A_{2} \leqq \cdots \leqq A_{n} \leqq \cdots$ be any non-decreasing sequence of positive numbers, and $\rho>0$. Then all statements (for all $\gamma$ )

$$
A_{n}=O\left(n^{\rho} \eta(\gamma / n)\right)
$$

are equivalent.

It is clear that if $\gamma<\gamma_{1}$, then $(2.1, \gamma)$ implies $\left(2.1, \gamma_{1}\right)$. If $\gamma>\gamma_{1}$, let $k$ be an integer such that $\gamma / \gamma_{1} \leqq k$. On replacing $n$ by $k n$ in $(2.1, \gamma)$ we have

$$
A_{n} \leqq A_{k n}=O\left((k n)^{\rho} \eta(\gamma / k n)\right)=O\left(n^{\rho} \eta\left(\gamma_{1} / n\right)\right),
$$

so that again $(2.1, \gamma)$ implies $\left(2.1, \gamma_{1}\right)$.

LEMMA 2.2. All statements

$$
\begin{gathered}
\sum_{\nu=1}^{\infty} \eta(\gamma / \nu)<\infty, \\
\sum_{\lambda=0}^{\infty} 2^{\lambda} \eta\left(\gamma 2^{-\lambda}\right)<\infty, \\
\int_{1}^{\infty} \eta(1 / t) d t<\infty
\end{gathered}
$$

are equivalent. It is understood that in $(2.2, \gamma),(2.3, \gamma), \gamma$ may assume independently any value $>0$, not necessarily the same in these two formulas.

First assume that $\gamma$ has the same value in $(2.2, \gamma),(2.3, \gamma)$. Since $\eta(\gamma / n) \downarrow 0$ as $n \uparrow \infty$ we have

$$
n \eta(\gamma / 2 n) \leqq \sum_{\nu=n+1}^{2 n} \eta(\gamma / \nu) \leqq n \eta(\gamma / n) .
$$

On putting here $n=2^{\lambda}$ and summing over $\lambda=0,1,2, \cdots$ we see at once that

\footnotetext{
$*$ We refer to [7] to the proofs of (i-iv).
} 
$(2.2, \gamma)$ is equivalent to $(2.3, \gamma)$. That $(2.2, \gamma)$ is equivalent to $(2.4)$ follows immediately from the Cauchy integral convergence test. Since however (2.4) does not depend on $\gamma$, we may give $\gamma$ independently any values in $(2.2, \gamma)$ and in $(2.3, \gamma)$, and all resulting statements will be equivalent.

\section{Lемма 2.3. All statements}

$$
\begin{aligned}
\sum_{\lambda=1}^{\infty} \eta\left(2^{-\lambda} \gamma / n\right) & =O(\eta(\gamma / n)) \text { as } n \rightarrow \infty, \\
\sum_{\nu=n}^{\infty} \nu^{-1} \eta(\gamma / \nu) & =O(\eta(\gamma / n)) \text { as } n \rightarrow \infty, \\
\int_{u}^{\infty} x^{-1} \eta(1 / x) d x & =O(\eta(1 / u)) \text { as } u \rightarrow \infty
\end{aligned}
$$

are equivalent, in the same sense as in Lemma 2.2.

Again we first assume $\gamma$ to be the same in $(2.4, \gamma)$ and $(2.5, \gamma)$. The equivalence of these two statements follows then immediately from the inequality

$$
\frac{1}{2} \eta(\gamma / 2 n) \leqq \sum_{\nu=n+1}^{2 n} \nu^{-1} \eta(\gamma / \nu) \leqq \eta(\gamma / n) .
$$

Since $\nu^{-1} \eta(\gamma / \nu)$ decreases when $\nu$ increases we may again apply the Cauchy test, with the result

$$
\begin{aligned}
\sum_{\nu=n}^{\infty} \nu^{-1} \eta(\gamma / \nu) & <n^{-1} \eta(\gamma / n)+\int_{n / \gamma}^{\infty} x^{-1} \eta(1 / x) d x \\
\int_{u}^{\infty} x^{-1} \eta(1 / x) d x & <\gamma^{-1} u^{-1} \eta(1 / u)+\int_{n / \gamma}^{\infty} x^{-1} \eta(1 / x) d x \\
& <\gamma^{-1} u^{-1} \eta(1 / u)+\sum_{\nu=n}^{\infty} \nu^{-1} \eta(\gamma / \nu)
\end{aligned}
$$

where, in the second formula, $n=[\gamma u]+1$. The first of these formulas shows that (2.6) implies $(2.5, \gamma)$, and the second that $(2.5, \gamma)$ implies $(2.6)$. Since (2.6) does not depend on $\gamma$, we complete the proof as in Lemma 2.2.

LEMMA 2.4. Let $\left\{a_{n}\right\}$ be any sequence of constants $a_{n} \geqq 0$, and $\rho>0$, then the statements

$$
\sum_{\nu=1}^{\infty}|\sin \nu t| \rho a_{\nu}=O(\eta(t)) \text { as } t \rightarrow 0
$$

and 


$$
n^{-\rho} \sum_{\nu=1}^{n} \nu^{\rho} a_{\nu}+\sum_{\nu=n+1}^{\infty} a_{\nu}=O(\eta(\pi / 2 n)) \text { as } n \rightarrow \infty
$$

are equivalent.

We first prove that (2.8) implies (2.7). Let (2.8) be satisfied and $t>0$ be given. Then on taking

$$
n=[\pi / 2 t], \quad n \leqq \pi / 2 t<n+1, \quad t>\pi / 2(n+1),
$$

we have

$$
\sum_{\nu=1}^{\infty}|\sin \nu t| \rho a_{\nu} \leqq t^{\rho} \sum_{\nu=1}^{n+1} \nu^{\rho} a_{\nu}+\sum_{\nu=n+2}^{\infty} a_{\nu}=O\left(\eta\left(\frac{\pi}{2(n+1)}\right)\right)=O(\eta(t)) .
$$

Conversely, let (2.7) be satisfied and a sufficiently large integer $n$ be given. Let $\delta>0$ be such that

$$
n \leqq \frac{\pi}{2 \delta}<n+1, \quad \frac{\pi}{2(n+1)}<\delta \leqq \frac{\pi}{2 n} .
$$

Since $\eta(t)$ increases when $t$ increases, an integration of $(2.7)$ over $(0, \delta)$ yields

$$
\sum_{\nu=1}^{\infty} a_{\nu} \int_{0}^{\delta}|\sin \nu t| \rho d t=O(\delta \eta(\delta)) \text {. }
$$

On the other hand, in view of our choice of $\delta$,

$$
\int_{0}^{\delta}|\sin \nu t|^{\rho} d t>(2 \nu / \pi)^{\rho} \int_{0}^{\delta} t^{\rho} d t>(\nu / 2)^{\rho} \delta^{\rho+1} /(\rho+1), \quad \nu=1,2, \cdots, n,
$$

and

$$
\begin{aligned}
& \int_{0}^{\delta}|\sin \nu t|^{\rho} d t=\nu^{-1} \int_{0}^{\nu \delta}|\sin t|^{\rho} d t \geqq \nu_{\delta} / \nu \int_{0}^{\pi / 2}(\sin t)^{\rho} d t \\
& >\nu_{\delta} / \nu \int_{0}^{\pi / 2}(\sin t)^{\rho} \cos t d t=\frac{\nu_{\delta}}{\nu(\rho+1)} \geqq \frac{\delta}{\pi(\rho+1)}, \quad \nu=n+1, \cdots,
\end{aligned}
$$

where

$$
\nu_{\delta}=\left[\frac{2 \nu \delta}{\pi}\right], \quad \nu_{\delta} \leqq \frac{2 \nu \delta}{\pi}<\nu_{\delta}+1, \quad \nu_{\delta} \geqq 1>\frac{2 \nu \delta}{\pi}-\nu_{\delta}, \quad \nu_{1}>\frac{\nu \delta}{\pi} .
$$

This gives

$$
\sum_{\nu=1}^{n}(\nu \delta) \rho a_{\nu}+\sum_{\nu=n+1}^{\infty} a_{\nu}=O(\eta(\delta))
$$


which is equivalent to (2.8) in view of (2.9).

LEMмa 2.5. Under the assumptions $\rho>0$ and

$$
\sum_{\lambda=1}^{\infty} \eta\left(2^{-\lambda} \gamma / n\right)=O(\eta(\gamma / n)) \text { as } n \rightarrow \infty, \quad \gamma>0 .
$$

all the statements

$$
\begin{aligned}
n^{-\rho} \sum_{\nu=1}^{n} \nu^{\rho} a_{\nu}+\sum_{\nu=n+1}^{\infty} a_{\nu} & =O(\eta(\gamma / n)) \text { as } n \rightarrow \infty, \\
n^{-\rho} \sum_{\nu=1}^{n} \nu^{\rho} a_{\nu} & =O(\eta(\gamma / n)) \text { as } n \rightarrow \infty, \\
\sum_{\nu=1}^{\infty}|\sin \nu t| \rho a_{\nu} & =O(\eta(t)) \quad \text { as } t \rightarrow 0
\end{aligned}
$$

are equivalent.

To prove that $(2.10, \gamma)$ and $(2.11, \gamma)$ are equivalent it suffices to prove that $(2.11, \gamma)$ implies $(2.10, \gamma)$, the converse being trivial. Put

$$
u_{0}=0, \quad u_{n}=\sum_{\nu=1}^{n} \nu^{\rho} a_{\nu}, \quad n=1,2, \cdots
$$

Then, if $(2.11, \gamma)$ is satisfied, we have

$$
\begin{aligned}
\sum_{\nu=n+1}^{2 n} a_{\nu} & =\sum_{\nu=n+1}^{2 n}\left(u_{\nu}-u_{\nu-1}\right) \nu^{-\rho} \\
& =-u_{n}(n+1)^{-\rho}+\sum_{\nu=n+1}^{2 n-1} u_{\nu}\left(\nu^{-\rho}-(\nu+1)^{-\rho}\right)+u_{2 n}(2 n)^{-\rho} \\
& =O(\eta(\gamma / n))+\sum_{\nu=n+1}^{2 n-1} O\left(\nu^{-1} \eta(\gamma / \nu)\right)+O(\eta(\gamma / 2 n))=O(\eta(\gamma / n)) .
\end{aligned}
$$

Consequently,

$$
\sum_{\nu=n+1}^{\infty} a_{\nu}=O\left(\sum_{\lambda=0}^{\infty} \eta\left(2^{-\lambda} \gamma / n\right)\right)=O(\eta(\gamma / n)),
$$

by $(2.4, \gamma)$. Thus $(2.10, \gamma)$ and $(2.11, \gamma)$ are equivalent. The proof of Lemma 2.5 is now readily completed by using Lemmas $2.1,2.3$, and 2.4 .

LEMMA 2.6. Let $a(u), b(u)$ be functions satisfying conditions

$$
0<\gamma \leqq a(u) \leqq 1, \quad 0<\gamma \leqq b(u) \leqq 1, \quad a(1)=b(1)=1,
$$

neither of them being identically equal to 1 . Then the following statements are equivalent: 


$$
\begin{aligned}
& \int_{1}^{u} \eta(1 / x) d x=O(u \eta(1 / u)) \text { as } u \rightarrow \infty \\
& \eta(1 / u)=\eta(1) a(u) u^{-1} \exp \left\{\int_{1}^{u} a(t) t^{-1} d t\right\} \\
& \int_{1}^{u} x \eta(1 / x)^{2} d x=O\left(u^{2} \eta(1 / u)^{2}\right) \text { as } u \rightarrow \infty, \\
& \eta(1 / u)=\eta(1) b(u) u^{-1} \exp \left\{\int_{1}^{u} b^{2}(t) t^{-1} d t\right\} .
\end{aligned}
$$

We first prove the equivalenceof (2.14) and (2.15). On putting $\phi(x)=\eta(1 / x)$ for $x \geqq 1$ and $\phi(x)=\phi(1)$ for $0<x<1$, we see that $\phi(x) \downarrow 0$ as $x \uparrow \infty$. Now if (2.14) is satisfied, we have

$$
u \phi(u) \leqq \int_{0}^{u} \phi(x) d x \leqq \gamma^{-1} u \phi(u),
$$

where necessarily $0<\gamma<1$. Put

$$
a(u)=\frac{u \phi(u)}{\phi_{1}(u)}, \quad \phi_{1}(u)=\int_{0}^{u} \phi(x) d x, \quad \phi_{1}(1)=\phi(1)=\eta(1) .
$$

Clearly $a(u)$ satisfies (2.13) while

$$
\frac{\phi_{1}^{\prime}(u)}{\phi_{1}(u)}=\frac{a(u)}{u}, \quad \phi_{1}(u)=\phi_{1}(1) \exp \left\{\int_{1}^{u} a(t) t^{-1} d t\right\} .
$$

On substituting this expression of $\phi_{1}(u)$ into $\phi(u)=a(u) u^{-1} \phi_{1}(u)$ we get (2.15). Thus (2.14) implies (2.15). The converse is readily proved by an easy computation by using (2.13). To prove the equivalence of (2.16) and (2.17) we apply an analogous argument starting from the inequalities

$$
\frac{1}{2} u^{2} \phi^{2}(u) \leqq \int_{0}^{u} x \phi^{2}(x) d x \leqq(2 \gamma)^{-1} u^{2} \phi^{2}(u)
$$

(implied by (2.16)) and putting presently

$$
\begin{aligned}
b^{2}(u) & =\frac{u^{2} \phi^{2}(u)}{2 \phi_{2}(u)}, \quad \phi_{2}(u)=\int_{0}^{u} x \phi^{2}(x) d x, \\
\phi_{2}^{\prime}(u) & =u \phi^{2}(u), \quad 2 b^{2}(u) \phi_{2}(u)=u \phi_{2}^{\prime}(u), \\
\phi_{2}^{\prime}(u) & =u \phi^{2}(u)=\phi^{2}(1) b^{2}(u) u^{-1} \exp \left\{2 \int_{1}^{u} b^{2}(x) x^{-1} d x\right\} .
\end{aligned}
$$


To complete the proof of our lemma it remains to show that (2.14) is equivalent to (2.16). On assuming (2.14) to be satisfied we have (2.15), whence

$$
\begin{aligned}
\int_{1}^{u} x \eta(1 / x)^{2} d x & <\eta(1)^{2} \int_{1}^{u} a(x) x^{-1} \exp \left\{2 \int_{1}^{x} a(t) t^{-1} d t\right\} d x \\
& =\frac{1}{2} \eta(1)^{2}\left[\exp \left\{2 \int_{1}^{u} a(t) t^{-1} d t\right\}\right]=O\left(u^{2} \eta(1 / u)^{2}\right)
\end{aligned}
$$

which is (2.16). Conversely on assuming (2.16) we have (2.17) so that

$$
\begin{gathered}
\eta(1 / u)>\eta(1) b^{2}(u) u^{-1} \exp \left\{\int_{1}^{u} b^{2}(x) x^{-1} d x\right\} \equiv \eta_{1}(1 / u), \\
\eta(1 / u)<\eta(1) \gamma^{-1} b^{2}(u) u^{-1} \exp \left\{\int_{1}^{u} b^{2}(x) x^{-1} d x\right\}=\gamma^{-1} \eta_{1}(1 / u) .
\end{gathered}
$$

The function $\eta_{1}(1 / u)$ clearly is of the type (2.15), hence satisfies (2.14), so that

$$
\int_{1}^{u} \eta(1 / x) d x<\gamma^{-1} \int_{1}^{u} \eta_{1}(1 / x) d x=O\left(u \eta_{1}(1 / u)\right)=O(u \eta(1 / u)) .
$$

The proof of Lemma 2.6 is now complete.

REMARK 2.1. Condition (2.14) of Lemma 2.6 is satisfied if, for a positive $\alpha<1, \eta(t) t^{-\alpha} \downarrow$ when $t \uparrow$. By Lemma 2.6, conditions (2.15), (2.16), (2.17) will be also satisfied in this case.

Indeed our assumption implies that $\eta(1 / x) x^{\alpha} \uparrow$ when $x \uparrow$, whence

$$
\int_{1}^{u} \eta(1 / x) d x \leqq u^{\alpha} \eta(1 / u) \int_{1}^{u} x^{-\alpha} d x=O\left(u \eta\left(1 / u^{\prime}\right)\right. \text {. }
$$

REMARK 2.2. If in (2.15)

$$
0<\gamma \leqq a(u) \leqq \gamma_{1}<1, \quad u>1,
$$

then

$$
\int_{1}^{\infty} x^{-1} \eta(1 / x) d x<\infty
$$

and

$$
\int_{t^{-1}}^{\infty} \tau^{-1} \eta(\tau) d \tau=O(\eta(t)) \text { as } t \rightarrow 0
$$


Conditions (2.19), (2.20) are also satisfied if for some positive $\alpha<1, \eta(t) t^{-\alpha} \downarrow$ when $t \downarrow 0$.

Indeed if (2.18) is satisfied then

$$
\eta(1 / u)=O\left(u^{-1} \exp \left\{\gamma_{1} \int_{1}^{u} t^{-1} d t\right\}\right)=O\left(u^{\gamma_{1}-1}\right) \text { as } u \rightarrow \infty,
$$

so that (2.19) holds. Again using the same notation as in the proof of Lemma 2.6 and assuming for simplicity $\eta(1)=1$ we have

Hence

$$
\begin{aligned}
\int_{t^{-1}}^{\infty} x^{-1} \eta(1 / x) d x & =\left.x^{-1} \phi_{1}(x)\right|_{t^{-1}} ^{\infty}+\int_{t^{-1}}^{\infty} x^{-2} \phi_{1}(x) d x \\
& =-\frac{\eta(t)}{a\left(t^{-1}\right)}+\int_{t^{-1}}^{\infty} \eta(1 / x) \frac{d x}{x a(x)} .
\end{aligned}
$$

$$
\int_{t^{-1}}^{\infty} x^{-1} \eta(1 / x)\left(\frac{1}{a(x)}-1\right) d x=\frac{\eta(t)}{a\left(t^{-1}\right)}=O(\eta(t)) \text { as } t \rightarrow 0 .
$$

Since, however,

$$
\frac{1}{a(x)}-1 \geqq \gamma^{-1}-1>0,
$$

we also have

$$
\int_{t^{-1}}^{\infty} x^{-1} \eta(1 / x) d x=O(\eta(t))
$$

which is (2.20). The last two statements of Remark 2.2 are readily proved if we observe that $\eta(1 / x) x^{\alpha} \downarrow$ as $x \uparrow$ implies (2.19) immediately while (2.20) follows then from

$$
\int_{t^{-1}}^{\infty} x^{-1} \eta(1 / x) d x<t^{-\alpha} \eta(t) \int_{t^{-1}}^{\infty} x^{-\alpha-1} d x=O(\eta(t)) \text { as } t \rightarrow 0 .
$$

3. Mean moduli of continuity. For any function $f(\theta)$ we shall use the notation

$$
\begin{aligned}
& \Delta_{0} f(\theta ; t)=f(\theta) \\
& \Delta_{1} f(\theta ; t)=f(\theta+t)-f(\theta-t) \\
& \Delta_{2} f(\theta ; t)=\Delta_{1} \Delta_{1} f(\theta ; t)=f(\theta+2 t)+f(\theta-2 t)-2 f(\theta) \\
& \Delta_{m} f(\theta ; t)=\Delta_{1} \Delta_{m-1} f(\theta ; t)=\sum_{\nu=0}^{m}(-1)^{\nu} C_{m, v} f(\theta+(m-2 \nu) t), \\
&
\end{aligned}
$$


If $f(\theta) \varepsilon L^{p}, p>0$ we define the expression

$$
\omega_{p}^{(m)}(f ; \delta)=\sup _{0 \leqq t \leqq \delta} M_{p}\left(\Delta_{m} f(\theta ; t) ;-\pi, \pi\right)
$$

as the mean modulus of continuity of order $m$ of $f(\theta)$ in $L^{p}$. We shall also write $\omega_{p}(\delta)$ or $\omega_{p}$ instead of $\omega_{p}^{(m)}(f ; \delta)$ if no confusion results. In view of $\S 1$, (iv), $\omega_{p}$ is an increasing function of $p$. It is clear that $\omega_{p}(\delta)$ is also an increasing function of $\delta$. Moreover it is well known that $M_{p}\left(\Delta_{1} f(\theta ; t)\right) \rightarrow 0$ as $t \rightarrow 0$ ("continuity in $L^{p}$ " of a function of $\left.L^{p}\right)$. The same is true then of $M_{p}\left(\Delta_{m} f(\theta ; t)\right)$ so that $\omega_{p}{ }^{(m)}(f ; \delta) \downarrow 0$ as $\delta \downarrow 0$. We also note that when $f(\theta)$ is continuous

$$
\lim _{p \rightarrow \infty} \omega_{p}^{(1)}(f ; \delta) \equiv \omega_{\infty}^{(1)}(f ; \delta)=\xi(f ; 2 \delta)
$$

(cf. $\$ 1,(1.2))$. Furthermore if

then

$$
f(\theta) \sim \sum_{\nu=-\infty}^{\infty} c_{\nu} e^{i \nu \theta},
$$

$$
\Delta_{m} f(\theta ; t) \sim \sum_{\nu=-\infty}^{\infty} c_{\nu}\left(e^{i \nu t}-e^{-i \nu t}\right)^{m} e^{i v \theta}
$$

With this notation we prove

LEMMA 3.1. If $p \geqq 1$, then

$$
2^{m}\left|c_{n}\right| \leqq \omega_{1}^{(m)}(\pi / 2 n) \leqq \omega_{p}^{(m)}(\pi / 2 n), \quad n=0,1,2, \cdots .
$$

We have

$$
c_{n}=\frac{1}{2 \pi} \int_{-\pi}^{\pi} f(\theta) e^{-i n \theta} d \theta=\frac{(-i)^{m}}{2 \pi} \int_{-\pi}^{\pi} f(\theta+(m-2 \nu) \pi / 2 n) e^{-i n \theta}(-1)^{\nu} d \theta,
$$

whence, by (3.1),

$$
2^{m}\left|c_{n}\right| \leqq \frac{1}{2 \pi} \int_{-\pi}^{\pi}\left|\Delta_{m} f(\theta ; \pi / 2 n)\right| d \theta \leqq \omega_{1}^{(m)}(\pi / 2 n) \leqq \omega_{p}^{(m)}(\pi / 2 n) .
$$

Theorem 3.1. Let $f(\theta) \varepsilon L^{p}, 1 \leqq p \leqq 2, k>0$, and

$$
\sum_{n=1}^{\infty} n^{-k / p^{\prime}}\left(\omega_{p}^{(m)}(\pi / 2 n)\right)^{k}<\infty . \dagger
$$

* For the case $m=1$, cf. $[19$, p. 18$]$.

$\dagger$ When $p=1$ we write

$$
\sum_{n=1}^{\infty}\left(\omega_{1}(m)(\pi / 2 n)\right)^{k}<\infty .
$$

The same remark refers to analogous situations in the sequel, without being stated explicity. 
Under these conditions

$$
\sum_{n=0}^{\infty}\left|c_{n}\right|^{k}<\infty
$$

For $p=1$ the theorem follows directly from (3.5). Now assume $p>1$. If $k=p^{\prime}$, the statement of the theorem is an immediate consequence of the YoungHausdorff inequality, without using assumption $(3.6, p)$. Hence the same will be true when $k>p^{\prime}$. Finally, let $0<k<p^{\prime}$. The Young-Hausdorff inequality, being applied to (3.4), gives

$$
\begin{aligned}
2^{m p^{\prime}} \sum_{\nu=-\infty}^{\infty}|\sin \nu t|{ }^{m p^{\prime}}\left|c_{\nu}\right|^{p^{\prime}} & =2^{m p^{\prime}+1} \sum_{\nu=1}^{\infty}|\sin \nu t|{ }^{m p^{\prime}}\left|c_{\nu}\right|^{p^{\prime}} \\
& \leqq M_{p}\left(\Delta_{m} f(\theta ; t)\right)^{p^{\prime}} \leqq \omega_{p}{ }^{(m)}(f ; t)^{p^{\prime}} .
\end{aligned}
$$

Hence, by Lemma 2.4,

$$
n^{-m p^{\prime}} \sum_{\nu=1}^{n} \nu^{m p^{\prime}}\left|c_{\nu}\right|^{p^{\prime}}+\sum_{\nu=n+1}^{\infty}\left|c_{\nu}\right|^{p^{\prime}}=O\left(\omega_{p}^{(m)}(\pi / 2 n)^{p^{\prime}}\right)
$$

and, a fortiori,

$$
n^{-m p^{\prime}} \sum_{\nu=1}^{n} \nu^{m p^{\prime}}\left|c_{\nu}\right|^{p^{\prime}}=O\left(\omega_{p}^{(m)}(\pi / 2 n)^{p^{\prime}}\right)
$$

On replacing $n$ by $2 n$ we have

$$
\sum_{\nu=n+1}^{2 n}\left|c_{\nu}\right|^{p^{\prime}}=O\left(\omega_{p}^{(m)}(\pi / 4 n)^{p^{\prime}}\right) .
$$

Now, by the Hölder inequality,

$$
\sum_{\nu=n+1}^{2 n}\left|c_{\nu}\right|^{k} \leqq n^{1 / q}\left(\sum_{\nu=n+1}^{2 n}\left|c_{\nu}\right|^{k q^{\prime}}\right)^{1 / q^{\prime}}, \quad q>1 .
$$

If we choose here

$$
k q^{\prime}=p^{\prime}, \quad q=p^{\prime} /\left(p^{\prime}-k\right)
$$

and combine with (3.9), we get

$$
\sum_{\nu=n+1}^{2 n}\left|c_{\nu}\right|^{k}=O\left(n^{1-k / p^{\prime}} \omega_{p}^{(m)}(\pi / 4 n)^{k}\right)=O\left(\sum_{\nu=n+1}^{2 n} \nu^{-k / p^{\prime}} \omega_{p}^{(m)}(\pi / 2 \nu)^{k}\right) .
$$

To complete the proof of Theorem 3.1 it remains to put $n=2^{\lambda}, \lambda=0,1, \cdots$ and take summation over $\lambda$. In view of $(3.6, p)$ this yields 


$$
\sum_{\nu=2}^{\infty}\left|c_{\nu}\right|^{k}=O\left(\sum_{\nu=2}^{\infty} \nu^{-k / p^{\prime}} \omega_{p}^{(m)}(\pi / 2 \nu)^{k}\right)<\infty .
$$

REMARK 3.1. From Lemma 2.2 we conclude that assumption $(3.6, p)$ of Theorem 3.1 can be replaced by any one of the equivalent assumptions

$$
\begin{gathered}
\sum_{n=1}^{\infty} n^{-k / p^{\prime}} \omega_{p}^{(m)}(\gamma / n)^{k}<\infty, \\
\sum_{\lambda=0}^{\infty} 2^{\lambda\left(1-k / p^{\prime}\right)} \omega_{p}^{(m)}\left(\gamma 2^{-\lambda}\right)^{k}<\infty, \\
\int_{1}^{\infty} x^{-k / p^{\prime}} \omega_{p}^{(m)}(1 / x)^{k} d x<\infty .
\end{gathered}
$$

REMARK 3.2. Note that a result of the preceding discussion is that any one of the conditions of Remark 3.1 imply (3.8).

The following theorem is a generalization of Theorem 3.1.

THEOREM 3.2. Let

$1 \leqq p \leqq 2,0<p_{1} \leqq p \leqq p_{2}, p=\rho_{1} p_{1}+\rho_{2} p_{2}, \rho_{1}+\rho_{2}=1, \rho_{1} \geqq 0, \rho_{2} \geqq 0$. If $f(\theta) \varepsilon L^{p_{2}}$ and if

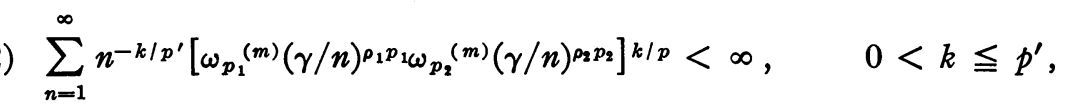

then

$$
\sum_{n=0}^{\infty}\left|c_{n}\right|^{k}<\infty .
$$

By the convexity property

$$
\omega_{p}^{p} \leqq \omega_{p_{1}}^{p_{1} p_{1}} \omega_{p_{2}}^{\rho_{2} p_{2}}
$$

and our theorem follows immediately from Theorem 3.1. In the case where $\omega_{p_{1}}{ }^{(m)}(\delta)<K \delta^{\alpha_{1}}, \omega_{p_{2}}{ }^{(m)}(\delta)<K \delta^{\alpha_{2}}, \alpha_{1}>0, \alpha_{2}>0$, [6, p. 252] condition (3.12) is satisfied whenever

$$
\alpha_{1} \rho_{1} p_{1}+\alpha_{2} \rho_{2} p_{2}>1+\left(\frac{1}{k}-1\right) p, \quad k \leqq p^{\prime} .
$$

With the notation $\rho_{1}=\rho, \rho_{2}=1-\rho, 0 \leqq \rho \leqq 1, p=\rho p_{1}+(1-\rho) p_{2}$ we get

$$
\alpha_{1} \rho p_{1}+\alpha_{2}(1-\rho) p_{2}>1+\left(\frac{1}{k}-1\right) p, \quad k \leqq p^{\prime}
$$


In particular, for $k=1$,

$$
\rho \alpha_{1} p_{1}+(1-\rho) \alpha_{2} p_{2}>1 .
$$

This condition for the case $m=1$ was given in a slightly different form by Hardy and Littlewood [6, p. 252]. For $p=2, p_{1} \leqq 2 \leqq p_{2}$, this condition reduces to

$$
\left(p_{2}-2\right) \alpha_{1} p_{1}+p_{1}>p_{2}-\left(2-p_{1}\right) \alpha_{2} p_{2} .
$$

Similarly, (3.13) reduces to

$$
\left(p_{2}-2\right) \alpha_{1} p_{1}+p_{1}>p_{2}-\left(2-p_{1}\right) \alpha_{2} p_{2}+2\left(\frac{1}{k}-1\right)\left(p_{2}-p_{1}\right) .
$$

If $\alpha_{1} p_{1}=1$ this becomes

$$
k>2\left(p_{2}-p_{1}\right) /\left\{2 p_{2}+\left(2-p_{1}\right) \alpha_{2} p_{2}-p_{1}-2\right\},
$$

which is less than 1 if $\alpha_{2} p_{2}>1$.

In the special case $\alpha_{1}=p_{1}=1$ we obtain the condition

$$
k>2\left(p_{2}-1\right) /\left\{\left(2+\alpha_{2}\right) p_{2}-3\right\} .
$$

Note that the class $\operatorname{Lip}_{1}(1,1)$ coincides with the class of functions of bounded variation $\left[4\right.$, p. $599 ; 5$, p. 619]. If, in addition, $f(\theta) \varepsilon \operatorname{Lip}\left(\alpha_{2}\right), p_{2}$ can be taken arbitrarily large, and so, on allowing $p_{2} \rightarrow \infty$, we get

$$
k>2 /\left(2+\alpha_{2}\right) \text {. }
$$

This result, in the case $m=1$, is due to Waraszkiewicz [16], while Zygmund [18, pp. 591-598; 19, p. 138] gave an example of divergence of $\sum\left|\rho_{n}\right|{ }^{k}$ for $k=2 /\left(2+\alpha_{2}\right)$. To summarize we state the

Corollary 3.1. Assume that $p_{1} \leqq 2 \leqq p_{2}$ and that

$\operatorname{Lip}_{m}(\alpha ; p) \quad\left\{\frac{1}{2 \pi} \int_{-\pi}^{\pi}\left|\Delta_{m} f(\theta ; t)\right|^{p} d \theta\right\}^{1 / p}<K t^{\alpha}, \quad t>0$,

for $\alpha=\alpha_{1}, p=p_{1}$ and $\alpha=\alpha_{2}, p=p_{2}$. The series $\sum_{0}^{\infty}\left|c_{n}\right|^{k}$ converges provided $k$ satisfies (3.13).

The basic inequality in the proof of Theorem 3.1 was (3.8). A partial converse is represented by the

THEOREM 3.3. Let $1 \leqq p \leqq 2$. Let $\eta(t)$, in addition to the general assumption $\eta(t) \downarrow 0$ as $t \downarrow 0$, be such that

$$
\sum_{\lambda=1}^{\infty} \eta\left(\frac{\pi}{n} 2^{-\lambda}\right)^{p}=O\left(\eta(\pi / n)^{p}\right) \text { as } n \rightarrow \infty .
$$


If the Fourier coefficients of $f(\theta)$ satisfy the condition

$$
\sum_{\nu=1}^{n} \nu^{m p}\left|c_{\nu}\right|^{p}=O\left(n^{m p} \eta(\pi / n)^{p}\right)
$$

then

$$
\omega_{p^{\prime}}^{(m)}(f ; t)=O(\eta(t)) \text { as } t \rightarrow 0 .
$$

For a given $t<\pi$ we have by the Young-Hausdorff inequality

$$
\begin{aligned}
M_{p^{\prime}}\left(\Delta_{m} f(\theta ; t)\right)^{p} & \leqq 2^{p m} \sum_{\nu=-\infty}^{\infty}|\sin \nu t|^{m p}\left|c_{\nu}\right|^{p} \\
& \leqq 2^{p m+1}\left\{\sum_{\nu=1}^{n}|\sin \nu t|^{m p}\left|c_{\nu}\right|^{p}+\sum_{\nu=n+1}^{\infty}\left|c_{\nu}\right|^{p}\right\}
\end{aligned}
$$

(cf. 1, (i)), where

$$
n=[\pi / t]+1>1, \quad \pi / n<t \leqq \pi /(n-1) .
$$

Let $S_{1}(t), S_{2}(t)$ denote the first and the second term in \{\} , respectively. Then, by (3.15),

$$
S_{1}(t) \leqq t^{m p} \sum_{\nu=1}^{n} \nu^{m p}\left|c_{\nu}\right|^{p}=O\left(\eta(\pi / n)^{p}\right)=O\left(\eta(t)^{p}\right) .
$$

Furthermore, if we replace $n$ by $2 n$ in (3.15) we readily find

$$
\sum_{\nu=n+1}^{2 n}\left|c_{\nu}\right|^{p}=O\left(\eta(\pi / n)^{p}\right)
$$

whence, by (3.14),

$$
S_{2}(t)=\sum_{\nu=n+1}^{\infty}\left|c_{\nu}\right|^{p}=O\left(\sum_{\lambda=1}^{\infty} \eta\left(\frac{\pi}{n} 2^{-\lambda}\right)^{p}\right)=O\left(\eta(\pi / n)^{p}\right)=O\left(\eta(t)^{p}\right) .
$$

Thus

$$
M_{p^{\prime}}\left(\Delta_{m} f(\theta ; t)\right)=O(\eta(t))
$$

which finally implies

$$
\omega_{p^{\prime}}^{(m)}(f ; t)=O(\eta(t)) .
$$

REMARK 3.3. From Lemmas 2.3 and 2.1 we conclude that assumption (3.14) of Theorem 3.3 can be replaced by any one of the equivalent assumptions $(\gamma>0)$

$$
\sum_{\lambda=1}^{\infty} \eta\left(2^{-\lambda} \gamma / n\right)^{p}=O\left(\eta(\gamma / n)^{p}\right) \text { as } n \rightarrow \infty \text {. }
$$




$$
\begin{aligned}
\sum_{\nu=n}^{\infty} \nu^{-1} \eta(\gamma / \nu)^{p} & =O\left(\eta(\gamma / n)^{p}\right) \text { as } n \rightarrow \infty, \\
\int_{u}^{\infty} x^{-1} \eta(1 / x)^{p} d x & =O\left(\eta(1 / u)^{p}\right) \text { as } u \rightarrow \infty,
\end{aligned}
$$

and the assumption (3.15) by the equivalent assumption

$$
(3.15, \gamma) \quad \sum_{\nu=1}^{n} \nu^{m p}\left|c_{\nu}\right|^{p}=O\left(n^{m p} \eta(\gamma / n)^{p}\right), \quad \gamma>0 \text { arbitrary. }
$$

In the special case $\eta(t)=K t^{\alpha} \log (1 / t)^{\beta}, 0<t<1,(3.14, \gamma)$ with $\gamma=1$ becomes

$$
\sum_{\lambda=1}^{\infty} n^{-\alpha} 2^{-\alpha \lambda}(\log n+\lambda \log 2)^{\beta}=O\left(n^{-\alpha}(\log n)^{\beta}\right)
$$

which is satisfied whenever $\alpha>0, \beta$ arbitrary. In the case $\alpha=1 / p, p>1, \beta=0$, $m=1$ this reduces to a theorem of Hardy and Littlewood [5, p. 620].

If $p=p^{\prime}=2$, the preceding discussion yields

THEOREM 3.4. Let $\eta(t)$, in addition to the property $\eta(t) \downarrow 0$ as $t \downarrow 0$, be such that

$$
\sum_{\lambda=1}^{\infty} \eta\left(2^{-\lambda} \frac{\gamma}{n}\right)^{2}=O\left(\eta(\gamma / n)^{2}\right) \text { as } n \rightarrow \infty
$$

\section{Then condition}

$$
\omega_{2}^{(m)}(f ; t)=O(\eta(t)) \text { as } t \rightarrow 0
$$

is necessary and sufficient in order that

$$
\sum_{\nu=1}^{n} \nu^{2 m}\left|c_{\nu}\right|^{2}=O\left(n^{2 m} \eta\left(\gamma^{\prime} / n\right)^{2}\right) \text { as } n \rightarrow \infty, \gamma^{\prime}>0 .
$$

4. Conjugate series.* The series

$$
\sum_{n=1}^{\infty}\left(a_{n} \sin n \theta-b_{n} \cos n \theta\right)
$$

is called the conjugate of

$$
a_{0} / 2+\sum_{n=1}^{\infty}\left(a_{n} \cos n \theta+b_{n} \sin n \theta\right) .
$$

* We refer to Zygmund [19] for various facts we use here of the theory of conjugate series and conjugate functions. 
If the latter is the Fourier series of a function $f(\theta) \varepsilon L^{p}, p>1$, then (4.1) is also the Fourier series of a function $\tilde{f}(\theta) \varepsilon L^{p}$, which is defined almost everywhere as the Cauchy principal of the integral

$$
\tilde{f}(\theta)=-\frac{1}{2 \pi} \int_{-\pi}^{\pi} f(t) \cot \frac{1}{2}(t-\theta) d t .
$$

THEOREM 4.1. If $p>1$ and

$$
\omega_{p}^{(m)}(f ; t)=O(\eta(t)) \text { as } t \rightarrow 0,
$$

then also

$$
\omega_{p}^{(m)}(\tilde{f} ; t)=O(\eta(t))
$$

The formulas

$$
\begin{aligned}
\tilde{f}(\theta) & =-\frac{1}{2 \pi} \int_{-\pi}^{\pi} f(\theta+\phi) \cot \phi / 2 d \phi, \\
\Delta_{m} \tilde{f}(\theta ; t) & =-\frac{1}{2 \pi} \int_{-\pi}^{\pi} \Delta_{m} f(\theta+\phi ; t) \cot \phi / 2 d \phi
\end{aligned}
$$

show that $\Delta_{m} \tilde{f}(\theta ; t)$ is conjugate to $\Delta_{m} f(\theta ; t)$. Hence by a theorem of M. Riesz [19], if $A_{p}$ denotes a constant depending only on $p$,

$$
M_{p}\left(\Delta_{m} \tilde{f}(\theta ; t)\right) \leqq A_{p} M_{p}\left(\Delta_{m} f(\theta ; t)\right)=O(\eta(t)) .
$$

This implies

$$
\omega_{p}^{(m)}(\tilde{f} ; t)=O(\eta(t)) .
$$

5. Inequalities for power series and polynomials. In the present section we state and prove some inequalities associated with power series and polynomials, which will be useful in constructing various "Gegenbeispiele" in subsequent sections.

Let

$$
F(z)=\sum_{n=0}^{\infty} c_{n} z^{n}, \quad z=r e^{i \theta}
$$

be a power series convergent for $0 \leqq r<1$. We write

$$
M_{p}(F ; r)=\left(\frac{1}{2 \pi} \int_{-\pi}^{\pi}\left|F\left(r e^{i \theta}\right)\right|^{p} d \theta\right)^{1 / p}, \quad p>0 .
$$

It is well known that $M_{p}(F ; r)$ is an increasing function of $r$ and that 
$\lim _{p \rightarrow \infty} M_{p}(F ; r) \equiv M_{\infty}(F ; r)=M(F ; r) \equiv \max _{|z| \leqq r}|F(z)|$.

LEMMA 5.1. Let $\chi(t)$ be a positive function,

$$
\chi(t / 2)=O(\chi(t)) \text { as } t \rightarrow 0,
$$

and $F(z)$ such that

$$
M_{p}(F ; r)=O\left((1-r)^{-\beta} \chi(1-r)\right) \text { as } \tau \rightarrow 1, \beta \geqq 0, p \geqq 1 .
$$

Then

$$
M_{q}(F ; r)=O\left((1-r)^{-\beta-1 / p+1 / q} \chi(1-r)\right), \quad p<q \leqq \infty .
$$

We first prove our lemma in the case $q=\infty$, when it reduces to

$$
M(F ; r)=O\left((1-r)^{-\beta-1 / p} \chi(1-r)\right) .
$$

We have

whence

$$
\begin{gathered}
F(z)=F\left(r e^{i \theta}\right)=\frac{1}{2 \pi} \int_{|\zeta|=\rho} \frac{F(\zeta) d \zeta}{\zeta-z}, \quad \zeta=\rho e^{i \tau}, \\
\rho=\frac{1+r}{2}, \quad 1-\rho=\rho-r=\frac{1-r}{2}
\end{gathered}
$$

$$
|F(z)| \leqq \frac{1}{2 \pi} \int_{-\pi}^{\pi} \frac{|F(\zeta)| d \tau}{\left|\rho e^{i(\theta-\tau)}-r\right|} \leqq M_{1}(F ; \rho) O\left((1-r)^{-1}\right) .
$$

Combining this with (5.2) for $p=1$ we see that

$$
M(F ; r)=O\left((1-r)^{-\beta-1} \chi(1-r)\right),
$$

which proves (5.4) in case $p=1$. If $p>1$, we have by the Hölder inequality

$$
\begin{aligned}
M(F ; r) & \leqq M_{p}(F ; \rho)\left\{\frac{1}{2 \pi} \int_{-\pi}^{\pi}\left|\rho e^{i \tau}-r\right|^{-p^{\prime}} d \tau\right\}^{1 / p^{\prime}} \\
& =M_{p}(F ; \rho) O\left\{\int_{0}^{\infty}\left[(\rho-r)^{2}+\tau^{2}\right]^{-p^{\prime} / 2} d \tau\right\}^{1 / p^{\prime}} \\
& =M_{p}(F ; \rho) O\left((1-r)^{-1 / p}\right)=O\left((1-r)^{-\beta-1 / p} \chi(1-r)\right) .
\end{aligned}
$$

The result in the case $p<q<\infty$ is now readily obtained from the convexity property combined with (5.4). Indeed, by (1.5),

$$
\begin{aligned}
M_{q}^{q} & \leqq M_{p}^{p} M^{q-p}=O\left((1-r)^{-\beta p} \chi(1-r)^{p}\right) O\left((1-r)^{-(q-p)(\beta+1 / p)} \chi(1-r)^{q-p}\right) \\
& =O\left((1-r)^{-\beta q+1-q / p} \chi(1-r)^{q}\right) .
\end{aligned}
$$

In the remaining lemmas of this section we shall use the following notation. 
Let

$$
F(z)=\sum_{\nu=0}^{\infty} c_{\nu} z^{\nu}
$$

converge for $r<1$. Let

$$
\sigma_{n} \equiv \sigma_{n}(z) \equiv \sigma_{n}(F ; z)=n^{-1}\left\{n c_{0}+(n-1) c_{1} z+\cdots+c_{n-1} z^{n-1}\right\}
$$

be the arithmetic mean of the $n$ first partial sums of the series (5.5). Let $\Omega(u)$ be a non-negative and convex function of $u \geqq 0, \Omega(0)=0$, so that $\Omega(u)$ is increasing. It is well known [19, pp. 83-84] that

$$
M_{1}\left[\Omega\left(\left|\sigma_{n}\right|\right)\right] \leqq M_{1}[\Omega(|F(z)|)] .
$$

In particular

$$
M_{p}\left(\sigma_{n}(z)\right) \leqq M_{p}(F(z)), \quad p \geqq 1 .^{*}
$$

We shall use these facts to prove

LEMMA 5.2. If a polynomial

$$
P_{n}(z)=c_{0}+c_{1} z+\cdots+c_{n} z^{n}, \quad z=e^{i \theta}
$$

satisfies the inequality

$$
M_{1}\left[\Omega\left(\left|P_{n}(z)\right|\right)\right] \leqq 1,
$$

then its derivative $P_{n}^{\prime}(z)$ satisfies

$$
M_{1}\left[\Omega\left(n^{-1}\left|P_{n}^{\prime}(z)\right|\right)\right] \leqq 1 . \dagger
$$

On writing

$$
P_{n}^{*}(z)=c_{n}+c_{n-1} z+\cdots+c_{0} z^{n}=z^{n} P_{n}(\bar{z}), \quad \bar{z}=e^{-i \theta}
$$

we have

$$
n \sigma_{n}\left(P_{n}^{*} ; z\right)=n c_{n}+(n-1) c_{n-1} z+\cdots+c_{1} z^{n-1}=z^{n-1} P_{n}^{\prime}(\bar{z}),
$$

whence, by (5.6),

$$
\begin{aligned}
\frac{1}{2 \pi} \int_{-\pi}^{\pi} \Omega\left[n^{-1}\left|P_{n}^{\prime}(z)\right|\right] d \theta & =\frac{1}{2 \pi} \int_{-\pi}^{\pi} \Omega\left[\left|\sigma_{n}\left(P_{n}^{*} ; z\right)\right|\right] d \theta \\
& \leqq \frac{1}{2 \pi} \int_{-\pi}^{\pi} \Omega\left[\left|P_{n}^{*}(z)\right|\right] d \theta=\frac{1}{2 \pi} \int_{-\pi}^{\pi} \Omega\left[\left|P_{n}(z)\right|\right] d \theta
\end{aligned}
$$

${ }^{*}$ Cf. $[9$, p. 333].

$\dagger$ Lemma 5.2 is a special case of a theorem of Zygmund [17, p. 394] which states the same result for any trigonometric polynomial instead of a polynomial in $e^{i \theta}$. We give a separate proof because of its simplicity. 
which is the desired result.

Corollary 5.1. If

$$
M_{p}\left(P_{n} ; r\right) \leqq 1, \quad 0 \leqq r=|z| \leqq 1, \quad p \geqq 1,
$$

then

$$
M_{p}\left(P_{n}^{\prime} ; r\right) \leqq n .
$$

For $r=1$ this is a special case of Lemma 5.2, corresponding to $\Omega(u)=u^{p}$. Since $M_{p}\left(P_{n} ; r\right), M_{p}\left(P_{n}^{\prime} ; r\right)$ are increasing functions of $r$, the result follows for any $r<1$. The limiting case of Corollary 5.1, as $p \rightarrow \infty$ is the classical theorem of M. Riesz [11]: If $\left|P_{n}(z)\right| \leqq 1,|z| \leqq 1$, then $\left|P_{n}^{\prime}(z)\right| \leqq n$. The proof above can be readily modified to yield this result also [13].

CoRollary 5.2. Let $\zeta=\rho e^{i t}, 0<\rho \leqq 1,0<t \leqq \pi / 2$ and

$$
Q(z)=\frac{P_{n}(z \zeta)-P_{n}(z \bar{\zeta})}{z(\zeta-\bar{\zeta})}, \quad z=r e^{i \theta}, \quad 0<r \leqq 1
$$

If (5.10) is satisfied, then

$$
M_{p}(Q ; r) \leqq n t / \sin t \leqq \frac{\pi}{2} n, \quad p \geqq 1
$$

We have

$$
P_{n}(z \zeta)-P_{n}(z \bar{\zeta})=z \int_{\bar{\zeta}}^{\zeta} P_{n}^{\prime}(z u) d u=i \rho z \int_{-t}^{t} P_{n}^{\prime}\left(z \rho e^{i \tau}\right) e^{i \tau} d \tau
$$

so that

$$
Q(z)=\frac{1}{2 \sin t} \int_{-t}^{t} P_{n}^{\prime}\left(z \rho e^{i \tau}\right) e^{i \tau} d \tau=\frac{t}{\sin t} I(z)
$$

where

$$
I(z)=\frac{1}{2 t} \int_{-t}^{t} P_{n}^{\prime}\left(z \rho e^{i \tau}\right) e^{i \tau} d \tau
$$

By the Minkowski inequality

$$
\left(\frac{1}{2 \pi} \int_{-\pi}^{\pi}|I(z)|^{p} d \theta\right)^{1 / p} \leqq \frac{1}{2 t} \int_{-t}^{t} d \tau\left[\frac{1}{2 \pi} \int_{-\pi}^{\pi}\left|P_{n}^{\prime}\left(z \rho e^{i \tau}\right)\right|^{p} d \theta\right]^{1 / p},
$$

whence

$$
M_{p}(Q ; r) \leqq \frac{t}{\sin t} M_{p}\left(P_{n}^{\prime} ; r \rho\right) \leqq \frac{t}{\sin t} n \leqq \frac{\pi}{2} n
$$


6. Converse theorems. In the present section we shall prove that various results of $\S 3$ are the "best possible," in a certain sense. We first prove a lemma which will be needed in the sequel.

LEMMA 6.1. If $\alpha_{\nu}>0, \beta_{\nu}>0, \alpha_{\nu} \downarrow 0$ as $\nu \uparrow \infty$, and $\sum_{\nu=0}^{\infty} \alpha_{\nu} \beta_{\nu}=\infty$, then

$$
\sum_{\nu=0}^{n}\left(\alpha_{\nu}-\alpha_{n+1}\right) \beta_{\nu} \rightarrow \infty \text { as } n \rightarrow \infty \text {. }
$$

For a given positive integer $m$ we can choose $n$ so large that $\alpha_{m}>2 \alpha_{n+1}$. Then $\alpha_{\nu}-\alpha_{n+1}>\frac{1}{2} \alpha_{\nu}, \nu=0,1, \cdots, m$, and

$$
\sum_{\nu=0}^{n}\left(\alpha_{\nu}-\alpha_{n+1}\right) \beta_{\nu}>\sum_{\nu=0}^{m}\left(\alpha_{\nu}-\alpha_{n+1}\right) \beta_{\nu}>\frac{1}{2} \sum_{\nu=0}^{m} \alpha_{\nu} \beta_{\nu} \rightarrow \infty \text { as } m \rightarrow \infty .
$$

Now to show that the result of Theorem 3.1 in the case $k=1$ is "the best possible" we shall prove the following:

THEOREM 6.1. Let $\eta(t)$, in addition to the property $\eta(t) \downarrow 0$ as $t \downarrow 0$, be such that

$$
\int_{1}^{u} \eta(1 / x) d x=O(u \eta(1 / u)) \text { as } u \rightarrow \infty
$$

while

$$
\sum_{u=1}^{\infty} n^{-1 / p^{\prime}} \eta(1 / n)=\infty, \quad 1 \leqq p \leqq 2
$$

Then there exists a function $f(\theta) \varepsilon L^{p}$ such that

$$
\omega_{p}^{(m)}(f ; \delta)<\eta(\delta) \text { but } \sum_{n=0}^{\infty}\left|c_{n}\right|=\infty .
$$

We introduce a sequence of polynomials

$$
g_{n}(z)=2^{-n\left(1+1 / p^{\prime}\right)}\left(\sum_{\nu=0}^{2^{n}} z^{\nu}\right)^{2}, \quad n=1,2, \cdots,
$$

a sequence of numbers $\epsilon_{n}=\eta\left(2^{-n}\right)$ and a sequence of positive integers $\left\{\lambda_{n}\right\}$ such that

$$
\lambda_{0}=0, \quad \lambda_{n}=2^{n+1}+n-2, \quad \lambda_{n+1}>\lambda_{n}+2^{n+1}, \quad n_{\perp}=0,1, \cdots
$$

Construct the power series

$$
G(z)=\sum_{\nu=1}^{\infty}\left(\epsilon_{\nu}-\epsilon_{\nu+1}\right) g_{\nu}(z) z^{\lambda \nu} \equiv \sum_{\nu=0}^{\infty} \gamma_{\nu} z^{\nu} .
$$


We shall establish the following properties of $G(z)$.

(i) $\gamma_{n}>0, n=0,1,2, \cdots$ and $\sum_{n=0}^{\infty} \gamma_{n}=\infty$.

(ii) $G(z)$ converges for $|z|<1$, and as $r \rightarrow 1, G\left(r e^{i \theta}\right)$ converges for almost all $\theta$ to a limit function

$$
G\left(e^{i \theta}\right) \equiv g(\theta) \varepsilon L^{p}, \quad g(\theta) \sim \sum_{n=0}^{\infty} \gamma_{n} e^{i n \theta} .
$$

(iii) $\omega_{p}^{(1)}(g ; \delta)=O(\eta(\delta))$ as $\delta \rightarrow 0$.

These properties clearly imply the truth of Theorem 6.1 if one observes that $\omega_{p}{ }^{(m)}(g ; \delta)=O\left(\omega_{p}{ }^{(1)}(g ; \delta)\right)$ as $\delta \rightarrow 0$. The fact that $\gamma_{n}>0$ follows immediately from the positiveness of the coefficients of $g_{n}(z)$. Furthermore

But

$$
\begin{aligned}
\sum_{\nu=0}^{\infty} \gamma_{\nu} & >\sum_{\nu=0}^{\infty}\left(\epsilon_{\nu}-\epsilon_{\nu+1}\right)\left(1+2+3+\cdots+2^{\nu}\right) 2^{-\nu\left(1+1 / p^{\prime}\right)} \\
& >\frac{1}{2} \sum_{\nu=0}^{\infty}\left(\epsilon_{\nu}-\epsilon_{\nu+1}\right) 2^{\nu\left(1-1 / p^{\prime}\right)} .
\end{aligned}
$$

(6.6) $\sum_{\nu=1}^{n}\left(\epsilon_{\nu}-\epsilon_{\nu+1}\right) 2^{\nu\left(1-1 / p^{\prime}\right)}=\sum_{\nu=1}^{n} \epsilon_{\nu} \beta_{\nu}-\epsilon_{n+1} 2^{n\left(1-1 / p^{\prime}\right)}=\sum_{\nu=1}^{n}\left(\epsilon_{\nu}-\epsilon_{n+1}\right) \beta_{\nu}$,

where

$$
\beta_{1}=2^{1-1 / p^{\prime}}, \quad \beta_{\nu}=2^{\nu\left(1-1 / p^{\prime}\right)}-2^{(\nu-1)\left(1-1 / p^{\prime}\right)}, \quad \nu=2,3, \cdots, n,
$$

and

$$
\sum_{\nu=1}^{n} \beta_{\nu}=2^{n\left(1-1 / p^{\prime}\right)}
$$

By Lemma 2.2, (6.2) is equivalent to the divergence of the series $\sum_{\nu=1}^{\infty} \epsilon_{\nu} 2^{\nu\left(1-1 / p^{\prime}\right)}$, so that the series

$$
\sum_{\nu=2}^{\infty} \epsilon_{\nu} \beta_{\nu}=\left(1-2^{-1+1 / p^{\prime}}\right) \sum_{\nu=2}^{\infty} \epsilon_{\nu} 2^{\nu\left(1-1 / p^{\prime}\right)}=\infty .
$$

By Lemma $6.1, \sum_{\nu=1}^{n}\left(\epsilon_{\nu}-\epsilon_{n+1}\right) \beta_{\nu} \rightarrow \infty$ as $n \rightarrow \infty$, whence $\sum_{n=0}^{\infty} \gamma_{n}=\infty$, and property (i) is proved. $G(z)$,

To prove (ii) denote by $u_{n}(z)$ the general term of the sum (6.5) defining

$$
u_{n}(z)=\left(\epsilon_{n}-\epsilon_{n+1}\right) g_{n}(z) z^{\lambda_{n}}
$$

We have 


$$
\begin{aligned}
M_{p}\left(u_{n}\left(e^{i \theta}\right)\right) & =\left(\epsilon_{n}-\epsilon_{n+1}\right) 2^{-n\left(1+1 / p^{\prime}\right)}\left(\frac{1}{2 \pi} \int_{-\pi}^{\pi}\left|\sum_{\nu=0}^{2^{n}} e^{i \nu \theta}\right|^{2 p} d \theta\right)^{1 / p} \\
& =\left(\epsilon_{n}-\epsilon_{n+1}\right) 2^{-n\left(1+1 / p^{\prime}\right)}\left(\frac{1}{\pi} \int_{0}^{\pi}\left|\frac{\sin \left(2^{n}+1\right) \theta / 2}{\sin \theta / 2}\right|^{2 p} d \theta\right)^{1 / p} \\
& <\left(\epsilon_{n}-\epsilon_{n+1}\right) 2^{-n\left(1+1 / p^{\prime}\right)} \pi^{2-1 / p}\left(\int_{0}^{\pi}\left|\frac{\sin \left(2^{n}+1\right) \theta / 2}{\theta}\right|^{2 p} d \theta\right)^{1 / p} \\
& <\left(\epsilon_{n}-\epsilon_{n+1}\right) 2^{-n\left(1+1 / p^{\prime}\right)} \pi^{2}\left(\int_{0}^{\infty}\left|\frac{\sin x}{x}\right|^{2 p} d x\right)^{1 / p}\left(\frac{1+2^{n}}{2}\right)^{2-1 / p} .
\end{aligned}
$$

Hence

$$
M_{p}\left(u_{n}(z)\right) \leqq M_{p}\left(u_{n}\left(e^{i \theta}\right)\right)<A\left(\epsilon_{n}-\epsilon_{n+1}\right), \quad z=r e^{i \theta},
$$

where

$$
A=\pi^{2}\left(\int_{0}^{\infty}\left|\frac{\sin x}{x}\right|^{2 p} d x\right)^{1 / p} \leqq \pi^{2}\left(\int_{0}^{\infty}\left(\frac{\sin x}{x}\right)^{4} d x\right)^{1 / 2}
$$

Thus

$$
\left|u_{n}(z)\right|<B\left(\epsilon_{n}-\epsilon_{n+1}\right)(1-r)^{-1 / p},
$$

where $B$ is a constant which does not depend on $n$ and the series defining $G(z)$ converges in $|z|<1$. Moreover, by the Minkowski inequality,

$$
M_{p}(G(z)) \leqq \sum_{n=1}^{\infty} M_{p}\left(u_{n}(z)\right) \leqq A \epsilon_{1} .
$$

This ensures the properties of $G(z)$ stated in (ii) (cf. F. Riesz [10]).

We now pass on to estimating $M_{p}\left(\Delta_{1} G(z ; t)\right)$. On putting $\zeta=e^{i t}$ we have for $r<1$,

$$
\Delta_{1} G(z ; t)=G\left(r e^{i(\theta+t)}\right)-G\left(r e^{i(\theta-t)}\right)=\sum_{\nu=1}^{\infty}\left\{u_{\nu}(z \zeta)-u_{\nu}(z \bar{\zeta})\right\}=\sum_{\nu=1}^{\infty} \Delta_{1} u_{\nu}
$$

Hence

$$
M_{p}\left(\Delta_{1} G(z ; t)\right) \leqq \sum_{\nu=1}^{\infty} M_{p}\left(\Delta_{1} u_{\nu}\right)=\sum_{\nu=1}^{n}+\sum_{\nu=n+1}^{\infty} \equiv S_{1}+S_{2} .
$$

From (6.7) we get immediately

$$
S_{2} \leqq 2 A \epsilon_{n+1} .
$$

The general term of $S_{1}$ can be estimated by means of Corollary 5.2 and (6.7), with the result 


$$
\begin{aligned}
S_{1} & \leqq 2 A t \sum_{\nu=1}^{n}\left(2^{\nu+1}+\lambda_{\nu}\right)\left(\epsilon_{\nu}-\epsilon_{\nu+1}\right) \leqq 10 A t \sum_{\nu=1}^{n} 2^{\nu}\left(\epsilon_{\nu}-\epsilon_{\nu+1}\right) \\
& \leqq 10 A t \sum_{\nu=1}^{n}\left(2^{\nu+1}-2^{\nu}\right) \epsilon_{\nu} \leqq 20 A t \int_{1}^{2^{n}} \eta(1 / x) d x=O\left(t 2^{n} \eta\left(2^{-n}\right)\right),
\end{aligned}
$$

by (6.1). Now assume $0<t<1$. If we choose $n$ so that $2^{n-1}<t^{-1} \leqq 2^{n}, n \geqq 1$, then

$$
S_{1}=O(\eta(t)), \quad S_{2}=O(\eta(t)),
$$

whence

$$
\omega_{p}^{(1)}(g ; \delta)=O(\eta(\delta)),
$$

and the proof of Theorem 6.1 is complete.

In the case $p=2$ it is possible to extend the result of Theorem 6.1 to hold for any $k>0$ as is shown by

Theorem 6.2. Let $\eta(t)$, in addition to the property $\eta(t) \downarrow 0$ as $t \downarrow 0$ be such that

$$
\int_{1}^{u} x \eta(1 / x)^{2} d x=O\left(u^{2} \eta(1 / u)^{2}\right) \text { as } u \rightarrow \infty,
$$

while, for a given $k, 0<k<2$,

$$
\sum_{n=1}^{\infty} n^{-k / 2} \eta(1 / n)^{k}=\infty \text {. }
$$

Then there exists a function $f(\theta) \varepsilon L^{2}$ such that

$$
\omega_{2}^{(m)}(f ; \delta)<\eta(\delta) \text { but } \sum_{n=0}^{\infty}\left|c_{n}\right|^{k}=\infty .
$$

The proof in many respects is similar to that of Theorem 6.1, so that we shall discuss in detail only the points where these two proofs differ from each other. We introduce the same sequence of polynomials $g_{n}(z)$ with $p^{\prime}$ replaced by 2 ,

$$
g_{n}(z)=2^{-3 n / 2}\left(\sum_{\nu=0}^{2^{n}} z^{\nu}\right)^{2}, \quad n=1,2, \cdots,
$$

and changing slightly the notation of Theorem 6.1 , construct the power series

$$
G(z)=\sum_{\nu=1}^{\infty} \alpha_{\nu} g_{\nu}(z) z^{\lambda \nu} \equiv \sum_{\nu=0}^{\infty} \gamma_{\nu} z^{\nu}, \quad \alpha_{\nu}=\left(\epsilon_{\nu}^{k}-\epsilon_{\nu+1}^{k}\right)^{1 / k},
$$

which reduces to the power series $G(z)$ of Theorem 6.1 for $k=1$. We shall 
establish the same properties (ii), (iii) which were proved for the $G(z)$ of Theorem 6.1, with $p$ replaced by 2 and the property

$$
\gamma_{n}>0, \quad \sum_{n=0}^{\infty} \gamma_{n}^{k}=\infty,
$$

instead of (i). As in the case of Theorem 6.1, Theorem 6.2 will follow from these properties.

To prove $(\mathrm{I}, k)$ we note again that $\gamma_{n}>0, n=0,1,2, \cdots$, while

$$
\sum_{\nu=0}^{\infty} \gamma_{\nu}^{k}>\sum_{\nu=1}^{\infty} \alpha_{\nu}^{k}\left(1+2^{k}+3^{k}+\cdots+2^{\nu k}\right) 2^{-3 \nu k / 2}>(k+1)^{-1} \sum_{\nu=1}^{n} \alpha_{\nu}^{k} 2^{\nu(1-k / 2)} \text {. }
$$

Property (I, $k$ ) will be proved if we show that

$$
\sum_{\nu=1}^{\infty} \alpha_{\nu}^{k} 2^{\nu(1-k / 2)}=\sum_{\nu=1}^{\infty}\left(\epsilon_{\nu}^{k}-\epsilon_{\nu+1}^{k}\right) 2^{\nu(1-k / 2)}=\infty .
$$

Since $\alpha_{\nu}^{k}=\epsilon_{\nu}^{k}-\epsilon_{\nu+1}^{k}$ this can be done in the same manner as in the proof of Theorem 6.1 , if we replace there $\eta$ by $\eta^{k}, \epsilon_{\nu}$ by $\epsilon_{\nu}^{k}, p^{\prime}$ by 2 , and $\beta_{\nu}$ by $2^{\nu(1-k / 2)}-2^{(\nu-1)(1-k / 2)}$ for $\nu>1$, and by $2^{1-k / 2}$ for $\nu=1$.

To prove property (ii), with $p$ replaced by 2 , we put

$$
u_{n}(z)=\alpha_{n} g_{n}(z) z^{\lambda_{n}}
$$

and prove, in the same manner as in the proof of Theorem 6.1, that

$$
M_{2}\left(u_{n}(z)\right)^{2}<A^{2} \alpha_{n}^{2} .
$$

Since

$$
\begin{aligned}
\alpha_{n}^{2}=\left(\epsilon_{n}^{k}-\epsilon_{n+1}^{k}\right)^{2 / k} & =\left(\epsilon_{n}^{k}-\epsilon_{n+1}^{k}\right)\left(\epsilon_{n}^{k}-\epsilon_{n+1}^{k}\right)^{2 / k-1} \\
& <\left(\epsilon_{n}^{k}-\epsilon_{n+1}^{k}\right) \epsilon_{n}^{2-k}<\epsilon_{n}^{2}-\epsilon_{n+1}^{2},
\end{aligned}
$$

we have $\sum_{\nu=1}^{\infty} \alpha_{\nu}^{2}<\infty$, and the remaining argument will be the same as in Theorem 6.1.

Finally, to prove property (iii) we now have

$$
M_{2}^{2}\left(\Delta_{1} G(z ; t)\right)=\sum_{\nu=1}^{\infty} M_{2}^{2}\left(\Delta_{1}\left(u_{\nu}\right)\right)=\sum_{\nu=1}^{n}+\sum_{\nu=n+1}^{\infty} \equiv S_{1}{ }^{(2)}+S_{2}{ }^{(2)} .
$$

In view of (6.13) and (6.14) we have

while

$$
S_{2}^{(2)}=O\left(\epsilon_{n+1}^{2}\right)
$$

$$
S_{1}^{(2)}=t^{2} O\left(\sum_{\nu=1}^{n}\left(\epsilon_{\nu}^{2}-\epsilon_{\nu+1}^{2}\right) 2^{2 \nu}\right)=t^{2} O\left(\sum_{\nu=1}^{n} \epsilon_{\nu}^{2}\left(2^{2 \nu}-2^{2 \nu-2}\right)\right)
$$




$$
=t^{2} O\left(\sum_{\nu=1}^{n} 2^{\nu} \epsilon_{\nu}^{2}\left(2^{\nu}-2^{\nu-1}\right)\right)=t^{2} O\left(\int_{1}^{2^{n}} x \eta(1 / x)^{2} d x\right) .
$$

On assuming $0<t<1 / 2$ and choosing $n$ so that

$$
2^{n}<t^{-1} \leqq 2^{n+1}, \quad n \geqq 1,
$$

we now have, in view of (6.9),

$$
\begin{aligned}
& S_{1}^{(2)}=O\left(t^{2} \int_{1}^{t^{-1}} x \eta(1 / x)^{2} d x\right)=O\left(\eta(t)^{2}\right), \\
& S_{2}{ }^{(2)}=O\left(\eta(t)^{2}\right),
\end{aligned}
$$

from which property (iii) is readily obtained.

REMARK 6.1. In view of Lemma 2.6 the conditions (6.1) of Theorem 6.1 and (6.9) of Theorem 6.2 are equivalent.

In the case $0<k \leqq 1$ the result of Theorem 6.2 can be improved as shows the following:

THEOREM 6.3. If conditions of Theorem 6.2 are satisfied with $0<k \leqq 1$, then there exists a continuous function $f(\theta)$ such that $\xi(f ; \delta)<\eta(\delta)$ but $\sum_{\nu=0}^{\infty}\left|c_{\nu}\right|^{k}=\infty$. The same result holds even when $1<k<2$, provided we make additional assumptions

$$
\int_{1}^{\infty} x^{-1} \eta(1 / x) d x=\int_{0}^{1} \tau^{-1} \eta(\tau) d \tau<\infty
$$

and

$$
\int_{t^{-1}}^{\infty} x^{-1} \eta(1 / x) d x=\int_{0}^{t} \tau^{-1} \eta(\tau) d \tau=O(\eta(t)) \text { as } t \rightarrow 0 .
$$

The proof is based on a result of one of our earlier papers [12]. We introduce a sequence of primes $\left\{q_{\nu}\right\}, q_{\nu} \equiv 1(\bmod 4)$ and such that for $\nu$ sufficiently large, $\nu>\nu_{0}$ say, each interval $\left(2^{\nu-1}, 2^{\nu}\right)$ would contain just one $q_{\nu}$. We set

$$
n_{\nu}=q_{1}+q_{2}+\cdots+q_{\nu}
$$

and observe that

$$
n_{\nu}=O\left(2^{\nu}\right)=O\left(q_{\nu}\right) \text { as } \nu \rightarrow \infty .
$$

For each $q_{\nu} \equiv q$ we put

$$
a_{j}^{(q)}=2 q^{-3 / 2}(q-j)\left(\frac{j}{q}\right), \quad j=1,2, \cdots, q-1,
$$


where $(j / q)$ is the Legendre symbol, and consider the polynomial

$$
g_{\nu}(z)=\frac{1}{2}\left(a_{q-1}^{(q)}+a_{q-2}^{(q)} z+\cdots+a_{1}^{(q)} z^{q-2}+a_{1}^{(q)} z^{q}+\cdots+a_{q-1}^{(q)} z^{2(q-1)}\right) .
$$

It is known (cf. [12]) that

$$
\left|g_{\nu}(z)\right| \leqq 1 \text { for }|z| \leqq 1
$$

Finally, let

$$
\begin{aligned}
u_{\nu}(z) & =\alpha_{\nu} q_{\nu}^{-1 / 2} z^{2 n^{\nu-1}-\nu+1} g_{\nu}(z), \\
\alpha_{\nu} & =\left(\epsilon_{\nu}^{k}-\epsilon_{\nu+1}^{k}\right)^{1 / k} 2^{\nu / 2}, \quad \epsilon_{\nu}=\eta\left(2^{-\nu}\right) .
\end{aligned}
$$

Note that for $0<k \leqq 1$,

$$
\left(\epsilon_{\nu}^{k}-\epsilon_{\nu+1}^{k}\right)^{1 / k}=\left(\epsilon_{\nu}^{k}-\epsilon_{\nu+1}^{k}\right)\left(\epsilon_{\nu}^{k}-\epsilon_{\nu+1}^{k}\right)^{1 / k-1} \leqq\left(\epsilon_{\nu}^{k}-\epsilon_{\nu+1}^{k}\right) \epsilon_{\nu}^{1-k} \leqq \epsilon_{\nu}-\epsilon_{\nu+1},
$$

and for $1<k<2$,

$$
\left(\epsilon_{\nu}^{k}-\epsilon_{\nu+1}^{k}\right)^{1 / k} \leqq \epsilon_{\nu}
$$

Thus

$$
\begin{aligned}
& \alpha_{\nu} \leqq 2^{\nu / 2}\left(\epsilon_{\nu}-\epsilon_{\nu+1}\right), \quad 0<k \leqq 1, \\
& \alpha_{\nu} \leqq 2^{\nu / 2} \epsilon_{\nu}, \quad 1<k<2,
\end{aligned}
$$

whence

(6.20) $\sum_{\nu=1}^{n} 2^{\nu / 2} \alpha_{\nu} \leqq\left\{\begin{aligned} & \sum_{\nu=1}^{n} 2^{\nu}\left(\epsilon_{\nu}-\epsilon_{\nu+1}\right) \leqq \sum_{\nu=1}^{n}\left(2^{\nu+1}-2^{\nu}\right) \epsilon_{\nu} \leqq 2 \int_{1}^{2^{n}} \eta(1 / x) d x, 0<k \leqq 1, \\ & \sum_{\nu=1}^{n} 2^{\nu} \epsilon_{\nu}=\sum_{\nu=1}^{n}\left(2^{\nu+1}-2^{\nu}\right) \epsilon_{\nu} \leqq 2 \int_{1}^{2^{n}} \eta(1 / x) d x, 1<k<2 .\end{aligned}\right.$

Similarly, for $0<k \leqq 1$,

$$
\sum_{\nu=n+1}^{\infty} 2^{-\nu / 2} \alpha_{\nu} \leqq \sum_{\nu=n+1}^{\infty}\left(\epsilon_{\nu}-\epsilon_{\nu+1}\right)=\epsilon_{n+1},
$$

while for $1<k<2$,

(6.22) $\sum_{\nu=n+1}^{\infty} 2^{-\nu / 2} \alpha_{\nu} \leqq \sum_{\nu=n+1}^{\infty} \epsilon_{\nu}=2 \sum_{\nu=n+1}^{\infty}\left(2^{\nu}-2^{\nu-1}\right) 2^{-\nu} \epsilon_{\nu}<2 \int_{2^{n}}^{\infty} x^{-1} \eta(1 / x) d x$.

Since $1 / q_{\nu}<2^{-\nu+1}$ for $\nu>\nu_{0}$, these formulas show that under assumptions of our theorem,

$$
\sum_{\nu=1}^{\infty} \alpha_{\nu} q_{\nu}^{-1 / 2}<\infty, \quad 0<k<2
$$


On the other hand for all $k, 0<k<2$ we have by (6.19), (6.20)

$$
\sum_{\nu=1}^{\infty} \alpha_{\nu}^{k} q_{\nu}^{1-k}>\frac{1}{2} \sum_{\nu=1}^{\infty}\left(\epsilon_{\nu}^{k}-\epsilon_{\nu+1}^{k}\right) 2^{\nu(1-k / 2)}=\infty .
$$

Now construct the power series

$$
G(z)=\sum_{n=1}^{\infty} u_{n}(z) \equiv \sum_{\nu=1}^{\infty} \gamma_{\nu} z^{\nu}
$$

Since

$$
\left|u_{n}(z)\right| \leqq \alpha_{n} q_{n}^{-1 / 2}
$$

formula (6.23) shows that $G(z)$ converges uniformly in $|z| \leqq 1$, hence is continuous in the closed unit circle, so that $g(\theta)=G\left(e^{i \theta}\right)$ is continuous. On the other hand $\gamma_{\nu}$ are the Fourier coefficients of $G(\theta)$ and we have

$$
\begin{aligned}
\sum_{\nu=1}^{\infty}\left|\gamma_{\nu}\right|^{k} & >\sum_{\nu=1}^{\infty} \alpha_{\nu}^{k} q_{\nu}^{-2 k}\left(1+2^{k}+3^{k}+\cdots+\left(q_{\nu}-1\right)^{k}\right) \\
& >(k+1)^{-1} \sum_{\nu=1}^{\infty} \alpha_{\nu}^{k} q_{\nu}^{-2 k}\left(q_{\nu}-1\right)^{k+1}>2^{-k-1}(k+1)^{-1} \sum_{i=1}^{\infty} \alpha_{\nu}^{k} q_{\nu}^{1-k}=\infty .
\end{aligned}
$$

To complete the proof of Theorem 6.3 it remains to estimate the modulus of continuity $\xi(g ; \delta)$ of $g(\theta)$. We have to estimate

$$
|g(\theta+t)-g(\theta)| \leqq \sum_{\nu=1}^{\infty}\left|u_{\nu}\left(e^{i(\theta+t)}\right)-u_{\nu}\left(e^{i \theta}\right)\right|=\sum_{\nu=1}^{n}+\sum_{\nu=n+1}^{\infty} \equiv S_{1}^{(0)}+S_{2}^{(0)} .
$$

In view of (6.26), (6.21), and (6.22) we see at once that

$$
S_{2}^{(0)} \leqq 2 \sum_{\nu=n+1}^{\infty} \alpha_{\nu} q_{\nu}^{-1 / 2}=O\left(\sum_{\nu=n+1}^{\infty} \alpha_{\nu} 2^{-\nu / 2}\right)
$$

whence

$$
\begin{aligned}
& S_{2}{ }^{(0)}=O\left(\epsilon_{n+1}\right)=O\left(\eta\left(2^{-n}\right)\right) \text { if } 0<k \leqq 1, \\
& S_{2}{ }^{(0)}=O\left(\int_{2^{n}}^{\infty} x^{-1} \eta(1 / x) d x\right)=O\left(\eta\left(2^{-n}\right)\right), \quad \text { if } 1<k<2 .
\end{aligned}
$$

To estimate $S_{1}{ }^{(0)}$ we use Corollary 5.2, (6.26), and (6.20) with the result

$$
S_{1}(0)=t O\left(\sum_{\nu=1}^{n} \alpha_{\nu} 2^{\nu / 2}\right)=t O\left(\int_{1}^{2^{n}} \eta(1 / x) d x\right), \quad 0<k<2 .
$$

Now assume $0<t<1$ and choose $n$ so that $2^{n-1}<1 / t \leqq 2^{n}$. Using (6.1) which 
is equivalent to assumption (6.9) of Theorem 6.2, and thus must be satisfied also in the case of Theorem 6.3, we have

$$
S_{1}{ }^{(0)}=O(\eta(t)), \quad S_{2}^{(0)}=O(\eta(t)) \text { as } t \rightarrow 0,
$$

and finally

$$
\xi(g ; t)=O(\eta(t)) .
$$

On multiplying $g(\theta)$ by a suitable constant factor we shall obtain a function $f(\theta)$ the existence of which is asserted in Theorem 6.3.

As an illustration of the preceding theory consider the function $\eta(t)=$ $t^{\alpha}(\log 1 / t)^{\beta}, 0<\alpha \leqq 1, \beta$ arbitrary real. Since

$$
\frac{d}{d t}\left(\eta(t) t^{-\rho}\right)=t^{\alpha-\rho-1}(\log 1 / t)^{\beta-1}\{(\alpha-\rho) \log (1 / t)-\beta\}
$$

$\eta(t) t^{-\rho} \downarrow 0$ as $t \downarrow 0$ if $0<\rho<\alpha$, and by remarks 2.1 and 2.2, $\eta(t)$ satisfies conditions (6.1), (6.15), and (6.16). Now

$$
\sum_{n=2}^{\infty} n^{-k / 2} \eta(1 / n)^{k}=\sum_{n=2}^{\infty} n^{-k / 2-\alpha k}(\log n)^{\beta k}<\infty
$$

if either $k>2 /(2 \alpha+1)$ and $\beta$ arbitrary, or $k=2 /(2 \alpha+1)$ and $\beta<-1 / k$ $=-(2 \alpha+1) / 2$. In this case, according to Theorem 3.1, condition $\omega_{2}^{(1)}(f ; \delta)$ $=O(\eta(\delta))$ implies $\sum_{n}\left|c_{n}\right|^{k}<\infty$.

If however $\beta=-1 / k=-(2 \alpha+1) / 2$, then the power series (6.25) for $z=e^{i 0}$ represents a continuous function $g(\theta)$ for which

$$
\xi(g ; \delta)=O\left(\delta^{\alpha} \log (1 / \delta)^{-\alpha-1 / 2}\right) \text { as } \delta \rightarrow 0,
$$

but

$$
\sum_{n}\left|\gamma_{n}\right|^{2 /(2 \alpha+1)}=\infty
$$

BIBLIOGRAPHY

1. S. BERNSTEIN. Sur la convergence absolue des séries trigonométriques. Comptes Rendus des Séances de l'Académie des Sciences, Paris, vol. 158 (1914), pp. 1661-1663.

2. - Same title. Communications de la Société Mathématique de Kharkoff, vol. 14 (1914), pp. 139-144 (in Russian).

3. - Same title. Comptes Rendus, vol. 199 (1934), pp. 397-400.

4. G. H. HARDy AND J. E. LitTLEwOoD. Some properties of fractional integrals. I. Mathematische Zeitschrift, vol. 27 (1928), pp. 565-606. II. Ibidem, vol. 34 (1932), pp. 403-439.

5. - A convergence criterion for Fourier series. Ibidem, vol. 28 (1928), pp. 612-634.

6. - Notes on the theory of series. IX. On the absolute convergence of Fourier series. Journal of the London Mathematical Society, vol. 3 (1928), pp. 250-253.

7. G. H. HaRdy, J. E. Littlewood, and G. Pólya. Inequalities. Cambridge, 1934.

8. E. Hille and J. D. TAMarkin. On the summability of Fourier series. III. Mathematische Annalen, vol. 108 (1933), pp. 525-576. 
9. K. NABEtani. Remarks on some theorems concerning sections of a power series. Tôhoku Mathematical Journal, vol. 41 (1935-36), pp. 333-336.

10. F. RIESz. Über die Randwerte einer analytischen Funktion. Mathematische Zeitschrift, vol. 18 (1923), pp. 87-95.

11. M. RrEsz. Eine trigonometrische Interpolationsformel und einige Ungleichungen fur Polynome. Jahresbericht der Deutschen Mathematiker-Vereinigung, vol. 23 (1914), pp. 354-368.

12. O. SzÁsz. Über den Konvergenzexponenten der Fourierschen Reihen gewisser Funktionenklassen. Sitzungsberichte der Bayerischen Akademie der Wissenschaften, München, 1922, pp. 135-150.

13. - Korlatos hatványsorokról, Matematikai és Természettudomanyi Ertesitö, vol. 43 (1926), pp. 504-520.

14. - Über die Fourierschen Reihen gewisser Funktionenklassen. Mathematische Annalen, vol. 100 (1928), pp. 530-536.

15. E. C. Titchmarsh. A note on Fourier transforms. Journal of the London Mathematical Society, vol. 2 (1927), pp. 148-150.

16. Z. WARASZkIEwICz. Sur un théorème de M. Zygmund. Bulletin International de l'Académie Polonaise, Classe A, Cracovie, 1929, pp. 275-279.

17. A. Zygmund. A remark on conjugate series. Proceedings of the London Mathematical Society, (2), vol. 34 (1932), pp. 392-400.

18. - Some points in the theory of trigonometric and power series. These Transactions, vol. 36 (1934), pp. 586-617.

19. - Trigonometrical Series. Warsaw-Lwów, 1935.

UNIVERSITY OF CincinNati,

Cincinnati, Ohio 